\title{
CómO FOMENTAR EL ESPÍRITU CRÍTICO EN LOS FUTUROS DOCENTES DE INGLÉS DESDE LA FORMACIÓN: FUNDAMENTOS Y PROPUESTAS
}

\author{
Fostering Critical Perspectives Among Future Teachers of English \\ L'ÉDUCATION INITIALE DES FUTURS ENSEIGNANTS D'ANGLAIS AVEC D'ESPRIT CRITIQUE: \\ DES PRINCIPES FONDAMENTAUX ET DES PROPOSITIONS
}

\author{
María Amor Barros-del Río \\ Doctora en Filología Inglesa, \\ Universidad de Burgos. Profesora \\ contratada doctora, Facultad de \\ Humanidades y Comunicación, \\ Universidad de Burgos. Paseo de \\ Comendadores $s / n$ (Hospital Militar) \\ 09001 Burgos, España. \\ abarros@ubu.es \\ https://orcid. \\ org/0000-0003-2537-7405
}

\begin{abstract}
RESUMEN
Las últimas décadas han sido testigos de la expansión del inglés como idioma internacional, un fenómeno íntimamente ligado a los procesos de globalización. Esta posición dominante hace difícil detectar los desequilibrios de poder que se esconden tras los usos lingüísticos, los mensajes que se transmiten o las comunidades de hablantes, entre otros aspectos. Igualmente, la enseñanza del inglés está impregnada de valores y políticas veladas que es preciso abordar desde la formación de los futuros docentes de lengua inglesa. Dado que a menudo el diseño y la implantación de dicha formación queda en manos de los docentes, se ha detectado que la formación inicial del profesorado desde una perspectiva crítica adolece de una fuerte fragmentación, por lo que es necesario integrar en una propuesta ordenada la enorme diversidad de iniciativas que existe al respecto. Este trabajo subraya la importancia de una pedagogía dialógica, una reflexión crítica y el abordaje de la identidad docente a través de una propuesta formativa categorizada e integral con el fin de que ese periodo formativo sea una herramienta útil, integral y transformadora que inculque el espíritu crítico en los futuros docentes de lengua inglesa.
\end{abstract}

Palabras clave: formación del profesorado; lengua inglesa; pensamiento crítico; pedagogía dialógica; reflexión crítica; identidad docente.

\section{Abstract}

The last decades have witnessed the expansion of English as an international language, a phenomenon intimately linked to the processes of globalization. This dominant position makes it difficult to detect the power imbalances that hide behind the linguistic uses, the messages that are transmitted, or the communities of speakers, among other aspects. Equally, the teaching of English is impregnated with veiled values and policies that must be addressed from the training of future teachers of English. Given that the design and implementation of such training is often in the hands of teachers, pre-service teacher training suffers from a strong

Recibido: 2018-03-08 / Aceptado: 2018-08-22 / Publicado: 2019-09-05

DOI: $10.17533 /$ udea.ikala.v24n03a06 
fragmentation. Thus, the enormous diversity of initiatives that exist needs to be integrated in an ordered and coherent proposal. This work emphasizes the importance of coupling a dialogic pedagogy with critical reflection and professional teacher identity within a categorized and integral formative proposal so that this formative period is a useful, integral and transforming tool to foster critical thinking in future English teachers.

Keywords: teacher training; English language; critical thinking; dialogic pedagogy; critical reflection; teacher identity.

\section{RÉSUMÉ}

Les dernières décennies ont vu l'expansion de l'anglais comme langue internationale, un phénomène intimement lié aux processus de la mondialisation. Cette position dominante rend difficile la détection des déséquilibres de pouvoir qui se cachent derrière les usages linguistiques, les messages transmis ou les communautés de locuteurs, entre autres aspects. De même, l'enseignement de l'anglais est imprégné de valeurs et de politiques voilées qui doivent être abordées à partir de la formation des futurs enseignants d'anglais. Étant donné que la conception et la mise en œuvre de cette formation sont souvent entre les mains des enseignants, il a été constaté que la formation des futurs enseignants d'un point de vue critique souffre d'une forte fragmentation. Donc, l'énorme diversité des initiatives existantes doit être intégrée dans une proposition ordonnée. Ce travail met l'accent sur l'importance d'une pédagogie dialogique dont la réflexion critique et l'identité des enseignants définissent une proposition de formation initiale catégorisée et complète pour que cette période soit utile et transformatrice pour encourager la pensée critique dans les futurs enseignants de la langue anglaise.

Mots-clés: formation initiale des enseignants; langue anglaise; pensée critique; pédagogie dialogique; réflexion critique; identité de l'enseignant. 


\section{Introducción}

En su conocida obra Pedagogía del oprimido, Paulo Freire (1970) realizaba una decidida defensa de la educación como instrumento liberador. Para él, la educación no solo ha de denunciar las desigualdades sociales, sino que también ha de ofrecerse como herramienta clave para empoderar a los oprimidos y ayudarlos a recuperar su dignidad a través de un proceso dialógico. Así pues, la pedagogía crítica pretende comprender e interrogar el contexto educativo y desarrollar prácticas pedagógicas que no solo transformen dicho contexto, sino también el resto de la sociedad (Pennycook, 1990). Para que esto sea posible, es preciso analizar la práctica educativa en los diversos elementos que la conforman, incluyendo los contenidos que se trabajan y los materiales que se utilizan, los roles del alumnado y del profesorado, y las instituciones educativas y sus políticas. Todas estas cuestiones son de especial aplicación a la enseñanza del inglés como lengua extranjera, ya que este idioma ha ido ganando terreno como medio de comunicación internacional en las últimas décadas. Se trata de un fenómeno íntimamente ligado a la globalización (Crystal, 2012; Philipson, 1992) que ha dado lugar a nuevas y numerosas comunidades de hablantes no nativos que utilizan el inglés de formas desiguales y en contextos diversos.

Este panorama mundial en el que el inglés se convierte en una lengua internacional queda claramente proyectado en el modelo de los círculos concéntricos propuesto por Braj Kachru (1985) y que desde su concepción ha derivado en un amplio ámbito de estudio conocido como World Englishes. La pluralidad y variedad de las comunidades parlantes existentes y sus usos ilustran la creciente complejidad de un fenómeno que a menudo queda oculto bajo el paraguas de la hegemonía cultural. Así, la enseñanza de idiomas se ha entendido a menudo como una fórmula sistemática y neutral para transmitir mensajes. Cuando el aprendizaje de un idioma se reduce a un propósito funcional, las relaciones de poder que enmarcan sus usos se detectan con menor facilidad, y pueden llegar a convertirse en un imperialismo lingüístico, tal y como advierte Philipson (1992).
Desde esta perspectiva dominante, se validan ciertas prácticas de enseñanza, se legitiman como universales solo algunas experiencias humanas y se orientan las interpretaciones adecuadas en cada caso, dejando sin presencia y sin voz a quienes Kumaravadivelu (2016) denomina subalternos, un colectivo que divide a los hablantes nativos y a los no nativos en una clara relación de desigualdad. Las críticas a las relaciones de poder intrínsecas en el uso del idioma inglés, que ponían en entredicho la supuesta neutralidad del lenguaje, comenzaron a dejarse oír en las últimas décadas del siglo xx (Fairclough, 1995; Kachru, 1985), pero no ha sido hasta más recientemente cuando la perspectiva crítica en la enseñanza de este idioma ha ido cobrando notoriedad (Crookes, 2013; Hawkins y Norton, 2009; Lin, 2004). Lo cierto es que tanto docentes como discentes a menudo no son conscientes de los desequilibrios de poder inherentes al aprendizaje de este idioma internacional, por lo que se hace necesaria una aproximación crítica al mismo.

Admitiendo que la enseñanza del inglés está impregnada de valores y políticas veladas, lo cierto es que este ámbito también ofrece grandes oportunidades para aumentar la conciencia crítica y la implementación de estrategias pedagógicas que la promuevan (Agray Vargas, 2010; Barnawi, 2010; Barrantes-Montero, 2014; Echeverri-Sucerquia, Arias y Gómez, 2014; Ghaemi y Taherian, 2011; Gounari, 2008; Norton y Toohey, 2004; Pennycook, 1990 y 2001). En este sentido, la formación de futuros docentes de inglés es sin duda una etapa privilegiada en la que el profesorado en formación puede familiarizarse con métodos y contenidos no convencionales y adquirir una visión crítica de su labor docente (Agray Vargas, 2007; Barrantes-Montero, 2014).

\section{La formación inicial del profesorado de lengua inglesa: una tarea pendiente de la pedagogía crítica}

La mejora de los programas de formación docente para la educación secundaria en España es una preocupación en el campo de la formación del profesorado (Escudero, 2009; Ruiz y Marcos, 1994). 
Los futuros docentes de lengua inglesa deben contar con un título de grado en Estudios Ingleses o disciplinas afines. Esta formación universitaria, que Jiménez Catalán (1997) ha calificado como muy pobre en competencias pedagógicas, ha de completarse con un máster profesionalizante a través del cual el alumnado se entrena en los fundamentos pedagógicos de la enseñanza. El máster de Profesor en Educación Secundaria Obligatoria y Bachillerato, Formación Profesional y Enseñanza de Idiomas es un requisito necesario para la incorporación a la actividad docente en los niveles señalados, según se estipula en la Ley Orgánica de Educación (Ley Orgánica 2/2006). Tanto la organización como la planificación general de este máster han de ajustarse a lo establecido en el Real Decreto 1393 - mediante el que se establece la planificación de las enseñanzas universitarias oficiales (Real Decreto 1393/2007) —, y en la Orden ECI - por la que se establecen los requisitos para la verificación de los títulos universitarios oficiales que habiliten para el ejercicio de las profesiones (Orden ECI 3859/2007) - para su posterior verificación. Sin embargo, la concreción de los contenidos que constituyen el currículo y la metodología que se va a utilizar quedan al arbitrio de cada universidad y, a la postre, del profesorado.

En términos generales, la enseñanza del inglés como lengua extranjera adolece de una orientación crítica, por lo cual queda muy a la zaga de otras disciplinas (Kumaravadivelu, 2006). Aunque la formación del profesorado de lengua extranjera sí ha visto avances en esta línea, especialmente en el ámbito internacional (Miller, Morgan y Medina, 2017), las propuestas se han orientado hacia las prácticas reflexivas (Shoffner, 2008), el compromiso social y cultural (Delano-Oriaran, 2014) y hacia el papel del docente de idiomas como agente transformador (Morgan, 2009, 2010), entre otros. Sin embargo, en el ámbito español sigue existiendo un debate abierto aún no resuelto y especialmente acuciante en los estudios de posgrado para la formación del profesorado, los cuales, según diversos autores, hacen especial hincapié en la profesionalización, la especificidad y la autonomía (Madrid, 2004; Montero y Vez, 1998), pero no abordan de forma explícita la necesidad de cultivar un espíritu crítico y las pedagogías que lo favorezcan. Por ello, es necesario hacer un llamamiento a los equipos docentes encargados de esta formación para que se replanteen, de forma sistemática y continua, en qué medida los programas formativos ofertados fomentan el espíritu crítico en los futuros docentes de lengua inglesa y para que, a la luz de sus conclusiones, incorporen mecanismos que garanticen esta necesidad.

En vista de la ausencia de modelos prescriptivos aceptados por la comunidad académica para la formación del profesorado de lengua inglesa con perspectiva crítica, será preciso adecuar los ámbitos de actuación concretos para ofrecer en cada contexto la formación que mejor promueva un espíritu crítico entre el futuro profesorado de lengua inglesa. A continuación, se exploran desde una perspectiva crítica algunos elementos necesarios de esa formación para que se convierta en una herramienta clave que inculque un espíritu crítico en la función docente.

\section{Una formación crítica para lograr un profesorado de inglés con espíritu crítico}

En la valiosa revisión que realizan Hawkins y Norton (2009) sobre las propuestas prácticas de formación crítica del profesorado, destacan tres orientaciones clave sobre las que pueden sustentarse los programas formativos para futuros profesores de lengua inglesa: promoción de una conciencia crítica, pedagogía dialógica y capacidad de autocrítica. Se trata de aspectos cuya naturaleza es menos obvia pero más poderosa que otro tipo de desafíos más visibles, como podrían ser el tamaño de los grupos, las limitaciones del tiempo, las necesidades propias de cada contexto, etc. Bajo estos tres ejes de carácter ideológico se agrupan cuestiones como propuestas de innovación curricular, la selección cuidadosa de contenidos, a veces incómodos y los correspondientes materiales y actividades, la gestión 
del aula, etc. Ninguno de estos elementos es más relevante que otro. Por el contrario, es deseable un enfoque integral de todos ellos para que respondan de forma óptima a los objetivos que se persiguen. A continuación, se desarrolla cada uno de estos ejes y se incluyen orientaciones prácticas.

\section{El fomento de una conciencia crítica}

No es posible adquirir una conciencia crítica si no se conoce la realidad. Y la realidad del mundo anglosajón es tan amplia como el propio planeta. A raíz de la desintegración del Imperio Británico, en todos los continentes surgen comunidades anglófonas, cuyas expresiones orales y escritas atienden a determinadas necesidades y contextos. Las múltiples formas en las que el inglés hoy está presente en las antiguas colonias británicas son también producto, en mayor o menor medida, de las influencias vernáculas. A esto hay que añadir el fenómeno de la globalización y la internacionalización del inglés como lengua de comunicación que ofrece una enorme variedad de intercambios y textos producidos por hablantes no nativos y utilizados entre ellos. La enormidad de esta realidad, cuyas interacciones trascienden los tres círculos, se ha constituido en objeto de estudio bajo el paraguas English as a Lingua Franca (Seidlhofer, 2013), así como sus implicaciones para la docencia (Jenkins y Leung, 2014).

Siendo este el panorama, los futuros docentes del inglés se enfrentan a una comunidad parlante nativa de unos 500 millones de personas a la que hay que sumar entre 1500 y 2000 millones de hablantes no nativos (Crystal, 2012). Esta enorme variedad, que Kachru (1985) agrupa en tres círculos concéntricos, incluye el círculo interior, que se restringe a los hablantes nativos; el exterior, que abarca aquellas antiguas colonias británicas y territorios en los que el inglés es una segunda lengua o lengua cooficial, y un tercer círculo en expansión, que incluye el resto de países en los que el inglés es utilizado para fines diversos. Esa variedad ha de ser objeto de estudio crítico por parte de docentes y aprendices. En esa medida, la formación del profesorado de lengua inglesa debe incluir no solo una perspectiva global y fundamentada de este fenómeno, sino también de sus múltiples manifestaciones concretas.

Dado que el contacto con el idioma puede estar máso menos limitado fuera del aula, los contenidos de una formación crítica han de basarse en la selección de materiales auténticos que favorezcan la reflexión crítica y el debate sobre las culturas que representan, ya que una pedagogía crítica implica no solo leer la palabra (word), sino también leer el mundo (world), como subrayaban Freire y Macedo (1987). Además, puesto que será el docente quien decida cuáles voces se escucharán y cuáles quedarán ocultas en el aula de inglés (Giroux, 1997; Lankshear y McLaren, 1993), será preciso esforzarse por abordar dinámicas y materiales provenientes no solo del círculo interior, sino también del exterior y del círculo en expansión en aras de una amplitud de registros que reflejen la realidad de la lengua en uso. Desde los fundamentos de la pedagogía crítica, la legitimación de los sin voz es una forma de resistir al imperialismo lingüístico y las fuerzas de poder que construyen desigualdades.

Esta perspectiva apoya la selección de materiales auténticos, aunque puedan ser problemáticos, ya que un currículum es transformador solo cuando el alumnado adquiere las estrategias necesarias para llegar a ser críticos (Giroux y McLaren, 1992). Algunas experiencias en esta línea han abordado la formación de los docentes tomando como eje vertebrador conocidas expresiones del círculo exterior, como el discurso de Malala ante las Naciones Unidas (Barros-del Río, 2016). En otros casos, la formación se ha realizado desde una aproximación intercultural que incluye viajes formativos (Walters, Garii y Walters, 2009), testimonios reales y materiales de lectura sobre interculturalidad (Agudelo, 2007). El uso de materiales audiovisuales, de fácil acceso a través de internet, es aconsejable especialmente si se tratan cuestiones de actualidad que permitan un posicionamiento crítico por parte de los futuros formadores. 
Además de materiales auténticos, un currículo para la formación crítica del profesorado debe incluir lecturas y documentos científicos avalados por la comunidad académica internacional (Gómez, Puigvert y Flecha, 2011). Sus contenidos pueden versar en torno a la evolución del inglés en el mundo, a la función docente y a la enseñanza del inglés como lengua extranjera. $\mathrm{La}$ lectura crítica de documentos sobre estos tres ejes temáticos, que vertebran la formación de los futuros profesores de inglés, tiene diversas funciones. Por una parte, actualiza la formación de los participantes y los familiariza con la investigación de vanguardia de modo que a su entrada en el mundo laboral estén en disposición de ofrecer a su alumnado información contrastada. Por otro lado, obliga a un esfuerzo intelectual individual primero, que luego, tras trabajarse de forma colaborativa, redunda en una mejor y mayor comprensión de lo leído. Por último, si la lectura es verdaderamente crítica, se supera la fase de comprensión del texto para llegar a identificar las ideas subyacentes, desafiar la ideología que las ordena y finalmente tomar postura tanto individual como colectivamente.

Como se puede apreciar, las posibilidades son muchas y la innovación curricular puede incluir prácticas, actividades y materiales diversos, así como temas conflictivos, aunque coincidimos con Lin (2008) en que "no habrá, y no debería haber, libros de recetas para presentar currículos críticos de lenguas, aparte de algunos principios y perspectivas generales que sirvan como guía para el educador crítico, y así adoptar y desarrollar pedagogías autóctonas para sus estudiantes" (p. 21).

\section{La práctica de una pedagogía dialógica}

Frente a la fórmula docente tradicional, unidireccional y autoritaria que para Freire (1970) era un instrumento más de opresión, la pedagogía crítica propone una práctica dialógica en la que tanto alumnado como profesorado sean agentes activos del aprendizaje a través de la puesta en práctica del pensamiento crítico. Del mismo modo, las metodologías que se recomienda utilizar en la formación del profesorado deben orientarse hacia esa praxis con un enfoque claramente colaborativo. Esto requiere, en primer lugar, asegurar un entorno de igualdad para que el diálogo sea liberador. El espacio que se utilice, la disposición de los participantes y la dinámica de las clases han de estar orientados a favorecer que el alumnado se exprese de forma igualitaria y democrática. Cuando se trabaja con todos los participantes a la vez, la colocación en círculo o semicírculo es idónea, pues permite un diálogo democrático. Como señalan Kosnik y Beck (2009), debates y círculos literarios son metodologías adecuadas para grupos grandes. Para organizar el trabajo en grupos más pequeños conviene disponer de equipamiento fácilmente movible que permita trabajar a través de centros de aprendizaje o proyectos grupales en forma simultánea, por ejemplo.

Asimismo, la propuesta freireana de una educación que plantee preguntas favorece la participación, la autonomía y la reflexión entre los futuros docentes, al tiempo que limita las intervenciones del formador favoreciendo una relación horizontal entre educador y educando (Shor, 1992). Sin embargo, esta dinámica no es suficiente si no va acompañada de una reflexión significativa, cuestión que ya desarrolló Edge (2011) al señalar la importancia de ser conscientes del proceso, de articular esa consciencia y de buscar el resultado en la experiencia vivida. Se trata, por tanto, de incorporar el pensamiento reflexivo a la experiencia formativa de modo que la labor docente futura cuente ya con la experiencia vivida de una formación consciente y crítica. En este sentido, no ha perdido vigencia la propuesta que hiciera Facione en 1992, la cual afirma que el pensamiento crítico debe incorporar capacidades cognitivas de interpretación, inferencia, regulación, evaluación, explicación y análisis que conducirían a la adecuada formulación de juicios. En concreto, el autor destaca tres estadios, a saber, interpretación o comprensión y expresión del significado de experiencias, datos, etc.; análisis, que engloba la identificación de relaciones de inferencia real entre distintos argumentos, conceptos, preguntas, opiniones, informaciones o creencias; y evaluación, que consiste en la valoración 
de la credibilidad de estas percepciones, ya sea a través de inferencias de relación lógica o mediante la propia experiencia. La suma de todos ellos daría como resultado un proceso cognitivo consciente, reflexivo y crítico. En esta línea, entre los instrumentos llevados a cabo que facilitan una actitud reflexiva se cuentan el uso de diarios personales tras las sesiones formativas (Chacón y Contreras, 2006; Lee, 2007; Zabalza, 1991), la observación por pares (Richards y Lockhart, 1991), la observación participante (Chacón, 2008) o la grabación en vídeo y posterior visualización con una actitud crítica (Orlova, 2009).

En última instancia, es necesario considerar la formación de los futuros docentes de acuerdo con el contexto en el que se desarrolla su profesión y de formas que superan la individualidad de cada docente. Al tiempo que se hace hincapié en la reflexión crítica personal y del grupo en formación, se han de establecer relaciones propositivas entre la labor educativa y su entorno más amplio, de modo que el tándem formación-acción pueda llevarse a cabo. Diversos estudios (Le Cornu y Ewing, 2008; Sierra, 2018) demuestran la idoneidad de las comunidades de aprendizaje como instrumento válido a largo plazo para una formación colegial y recíproca del profesorado, especialmente en los periodos de prácticas.

\section{La conciencia crítica de sí mismo}

En la formación del futuro profesorado de inglés hay algunos aspectos que a menudo se soslayan y que solo en los últimos años han ido suscitando interés. Nos referimos a los procesos de construcción de la identidad docente, cuestión de difícil abordaje ya que pertenece en gran medida al ámbito de lo subjetivo. Sin embargo, se trata de un tema esencial que, si bien desborda el periodo formativo incluyendo también la vida profesional docente, encuentra sus fundamentos y motivaciones en esta etapa inicial.

Frente al discurso dominante que favorece al docente nativo de lengua inglesa frente al no nativo, incluso en la selección profesional (Clark y Paran, 2007), los futuros docentes de inglés, cuya lengua materna no es esa no pueden evitar cuestionarse su capacidad en el desempeño de la profesión, como algunos estudios han puesto de manifiesto (Canagarajah, 1999; Mahboob, 2010; Medgyes, 1992). Esto ocurre porque ideológicamente la propiedad del lenguaje y el prestigio siguen estando reducidos a los habitantes de los países del círculo interior, a pesar de ser muy superior el número de hablantes de los círculos exterior y en expansión. Por ello, es preciso que la formación del profesorado incluya fórmulas pedagógicas y contenidos curriculares que construyan identidades profesionales positivas, tal y como han propuesto Kamhi-Stein (1999) y Llurda (2006), entre otros.

En un primer momento, es esencial tener en cuenta las experiencias previas del alumnado. Su condición de discentes del idioma a lo largo de más o menos años de estudio los sitúa en una posición única que abarca, no solo su historial académico, sino también las experiencias personales que de dicha formación se deriven. Tanto si estas han sido positivas e inspiradoras, como si en ellas aprecian carencias, limitaciones y frustraciones, es preciso reconocer que son parte de esa identidad en construcción.

Coincidimos con Paulo Freire en que una pedagogía de la autonomía debe atender tanto al conocimiento existente como al conocimiento aún no existente (Freire, 2006). Por ello, para una formación significativa y eficaz, será necesario que el formador o formadora domine el conocimiento y las experiencias previas de los futuros docentes, y que el propio alumnado re-conozca su valía como punto de partida sobre el que seguir construyendo individual y colectivamente ese proyecto vital que es la labor docente. Una encuesta inicial que incluya preguntas abiertas sobre tres grandes ejes: la experiencia previa como discentes, las expectativas sobre la futura labor docente y los contenidos más relevantes para la docencia del inglés como lengua extranjera ofrecerá una imagen fotográfica sobre las características del grupo, sus deficiencias y anhelos, así como sobre el conocimiento existente que el propio grupo aportará al desarrollo del curso. Los resultados recogidos 
obligarán, por otra parte, a que el formador o formadora mantenga un espíritu abierto y flexible, de manera que cada curso académico se acomode a las necesidades concretas del alumnado. El desafío, en este caso, es partir de los conocimientos del profesorado en formación y seguir construyendo con base en ellos.

$\mathrm{Al}$ mismo tiempo, esta dinámica obliga a los futuros profesionales a la reflexión personal y a la conciencia de sí mismos (Jenson, 2011). Si a la realización de la encuesta inicial y a la posterior selección de los aspectos más coincidentes les sigue una exposición de los mismos, el grupo puede conseguir iniciar el proceso hacia la autonomía crítica que tan útil resultará en el curso de la función docente. Este camino encontrará otro punto de inflexión en la encuesta final en la que el alumnado podrá evaluar la adecuación de los contenidos y la metodología implementada, así como el proceso de emancipación crítica que la formación recibida haya podido provocar en cada uno de los participantes.

A continuación, es necesario abordar la cuestión de la identidad como docentes de inglés. En este sentido, la primera tarea es desterrar el mito del profesor/hablante nativo, puesto que como hemos visto la hegemonía cultural sigue identificando la propiedad del idioma con los hablantes del círculo interior (Norton, 1997). La fortaleza de esta construcción ideológica deviene de una gran diversidad de recursos que incluyen materiales didácticos escritos y audiovisuales, normas, usos y expresiones lingüísticas, instituciones de enseñanza de prestigio que avalan los niveles alcanzados en el idioma, empresas internacionales de inmersión lingüística, y un sinfín de fórmulas de aprendizaje a menudo vinculadas a un beneficio económico. Además, el inglés, en muchos casos, es un negocio que confirma la supremacía de los hablantes del círculo interior frente al resto de angloparlantes. Por ello, es esencial que la formación de los futuros docentes incluya la adopción libre y consciente de posturas políticas y pedagógicas que les permitan situarse tanto en su propia realidad como en la relación intercultural que sin duda se verán obligados a encarar (Siqueira, 2017).
Ante esta circunstancia, es preciso detectar las relaciones de poder subyacentes al concepto de identidad y provocar la negociación de dicha identidad entre los futuros profesionales (Pavlenko y Blackledge, 2004). Para empezar, conviene trabajar de forma reflexiva las falacias inherentes al hablante nativo que Canagarajah (1999) desglosa desde la lingüística, la política, la economía y la labor docente. En particular, será deseable cuestionar la superioridad del hablante nativo como profesional docente, una falacia ampliamente extendida que tiende a dejar en la sombra la importancia de una buena preparación pedagógica y experiencia práctica, al tiempo que ensalza la supuesta excelencia docente de la lengua materna por el mero hecho de hablar dicha lengua. Luego será acertado presentar experiencias de docentes no nativos de lengua inglesa (Park, 2012) y ahondar en esta realidad desde la experiencia propia y los mitos existentes, profundizando en los mitos comúnmente aceptados, los ejemplos concretos en los que esto se evidencia, y la reflexión crítica ante dichos episodios. Toda esta labor debe conducir, finalmente, a planteamientos propositivos de los participantes, que ayuden a reconstruir una identidad docente basada en el reconocimiento, e incluso en el orgullo, de no ser nativo/a del idioma que se va a enseñar. La competencia multilingüe, como señala Canagarajah, puede llegar a ser una ventaja a la hora de enseñar una lengua extranjera, pues hace más eficaces a estos formadores (1999, p. 80).

Un último apunte debe dirigirse a la educación de los formadores. Se trata de un aspecto necesario para que las actitudes previamente señaladas puedan desarrollarse. La necesidad de reafirmación y concienciación de la enseñanza del inglés que propenda por la igualdad y el empoderamiento se encontrará, demasiado a menudo, escasa e incluso ausente en los espacios formativos. De esta manera, habrá de exigirse dichos espacios a las autoridades pertinentes, o bien serán el fruto de la organización tenaz y de la autogestión de los formadores. En el Estado español no se han encontrado espacios organizados para la formación crítica de docentes exclusivamente en el área lingüística y del ámbito universitario, a 
excepción de la asociación FEDICARIA, que desde 1991 aglutina a profesores de universidad, enseñanzas medias y enseñanza primaria, sobre todo en las disciplinas de las ciencias sociales. ${ }^{1}$ Esta plataforma de trabajo intelectual cuestiona cómo entender y hacer efectivos los postulados del pensamiento crítico en su ámbito de docencia.

\section{Conclusiones}

En las últimas décadas, la lengua inglesa ha sufrido un desarrollo exponencial a nivel mundial transcendiendo el ámbito de las relaciones comerciales para convertirse en el idioma de la globalización. Sin embargo, estas transformaciones aún no se reflejan en la formación del futuro profesorado en lengua inglesa que, en el caso español, y con seguridad de otros muchos países, sigue descontextualizada y a merced del criterio de los equipos docentes de turno.

Lejos de plantear un marco hermético para esa formación inicial del profesorado desde una perspectiva crítica, este trabajo propone una reflexión en torno al diseño y la puesta en práctica de los programas formativos actuales del estado español, ofreciendo líneas de trabajo que deriven en una diversidad de propuestas transformadoras. Se trata de una proposición abierta a los equipos docentes para que replanteen los programas formativos orientándolos al cultivo del pensamiento crítico entre los futuros docentes de lengua inglesa. Para ello, se han articulado diversas alternativas en torno a los tres ejes señalados por Hawkins y Norton (2009), que giran en torno a la promoción de una conciencia crítica, al uso de una pedagogía dialógica y al fomento de la capacidad de autocrítica. Como se ha señalado, no se trata simplemente de realizar un nuevo diseño curricular ni de ejecutar pedagogías dialógicas y estrategias colaborativas ni tampoco de enfrentar al alumnado en formación consigo mismo o con las injusticias del mundo para que tome conciencia. El objetivo es mucho más ambicioso: se trata de generar la necesidad de una formación integral crítica, consciente,

1 Para más información, se aconseja visitar la página http://www.fedicaria.org transformadora y permanente entre el futuro profesorado de lengua inglesa. Desde el punto de vista pedagógico, la adquisición de una conciencia crítica a través del conocimiento sólido del fenómeno anglófono en sus dimensiones internacional y local, serán aspectos irrenunciables que habrán de acompañarse de una innovación curricular que seleccione materiales auténticos, incluso si estos son "incómodos”, y ponga en práctica pedagogías colaborativas, dialógicas y reflexivas. Además, desde la dimensión comprometida del ser docente en un mundo en cambio, el diálogo intercultural, la familiaridad con la investigación de vanguardia y la conciencia de uno mismo como profesional no nativo de la lengua que se enseña, la formación de los futuros docentes excederá, sin duda, el periodo preprofesional, y habrá de adquirir una dimensión longitudinal que se mantenga a lo largo de la carrera docente.

Hasta ahora, las iniciativas llevadas a cabo han incidido en algunos de los aspectos señalados, pero en términos generales adolecen de la unidad y coherencia que exige la formación de personas y profesionales que desarrollen su labor docente con la mirada puesta en la justicia.

Queda en el tintero el abordaje del periodo de prácticas en centros educativos, una etapa esencial para el profesorado en formación y que a menudo se ha visto relegada a un segundo plano desde los círculos académicos. Integrar ideológica y pedagógicamente todo el periodo formativo, y más allá, desde una perspectiva crítica, es un desafío al que aún no se ha dado respuesta y que habrá de tratarse en futuros trabajos.

Hoy más que nunca es necesario formar educadores de lengua inglesa que de forma realista, crítica, informada y ética formen a su vez nuevas generaciones con espíritu crítico, pues como señala Giroux (1997), solo a través de la vivencia de una formación liberadora podrán los futuros docentes ejercer su profesión en la misma clave y convertirse en intelectuales transformadores. El desafío, que sin duda transciende lo profesional, queda ahí. Con este trabajo esperamos inspirar a los equipos de formadores en esta tarea tan compleja y a la vez tan motivadora. 


\section{Referencias}

Agray Vargas, N. (2010). La construcción de currículo desde perspectivas críticas: una producción cultural. Signo y Pensamiento, 29(56), 420-427.

Agray Vargas, N. (2007). Currículo emancipatorio y enseñanza de lenguas extranjeras. ¿Ficción o realidad? GIST: Education and Learning Research Journal, 1, 115-124.

Agudelo, J. J. (2007). An intercultural approach for language teaching: Developing critical cultural awareness. Íkala, Revista de Lenguaje y Cultura, 12(18), 185-217.

Barnawi, O. Z. (2010). Pedagogical tasks for fostering critical transformative EFL teachers: pre-service EFL teacher-educators. The Journal of International Social Research, 3(4), 107-119.

Barrantes-Montero, L. G. (2014). La teoría del decrecimiento y el proceso de enseñanza-aprendizaje de lenguas. Revista Electrónica Educare, 18(3), 251-261. https:// doi.org/10.15359/ree.18-3.15

Barros-del Río, M. A. (2016). Promoting critical awareness in Spanish pre-service ELT training: A chance to change the world. International Journal of English and Education, 4(3), 252-263.

Canagarajah, A. S. (1999). Interrogating the "native speaker fallacy": Non-linguistic roots, non-pedagogical results. En G. Braine (Ed.), Non-native educators in English language teaching (pp. 77-92). Nueva York y Londres: Lawrence Erlbaum.

Chacón, M. A. (2008). Las estrategias de enseñanza reflexiva en la formación inicial docente. Educere, 12(41), 277-287.

Chacón, M. y Contreras, A. (2006). Los diarios de prácticas: una estrategia de reflexión en la formación docente. Acción Pedagógica, 15, 120-127.

Clark, E. y Paran, A. (2007). The employability of nonnative-speaker teachers of EFL: A UK survey. System, 35(4), 407-430. https://doi.org/10.1016/j. system.2007.05.002

Crookes, G. V. (2013). Critical ELT in action. Foundations, promises, praxis. Nueva York: Routledge. https:// doi.org/10.4324/9780203844250

Crystal, D. (2012). A global language. En P. Seargeant y J. Swann (Eds). English in the world: History, diversity, change (pp. 152-157). Abingdon: Routledge. https://doi.org/10.1017/CBO9781139196970

Delano-Oriaran, O. (2014). Engaging pre-service teachers in diverse communities through service-learning: a practical guide for application. Journal of Education for Teaching, 40(2), 186-188. https://doi.org/10.10 $80 / 02607476.2013 .869968$

Echeverri-Sucerquia, P. A., Arias, N., y Gómez, I. C. (2014). La pedagogía crítica en la formación de docentes de inglés: La experiencia de un grupo de estudio. Íkala, Revista de Lenguaje y Cultura, 19(2), 167-181. https://doi.org/10.17533/udea.ikala.v19n2a04

Edge, J. (2011). The reflexive teacher educator in TESOL: Roots and wings. Nueva York: Routledge. https:// doi.org/10.4324/9780203832899

Escudero, J. M. (2009). La formación del profesorado de educación secundaria: contenidos y aprendizajes docentes. Revista de Educación, 350, 79-103.

Facione, P. A. (1992). Critical thinking: What it is and why it counts. Insight Assessment, 1-28. Disponible: https:// www.nyack.edu/files/CT_What_Why_2013.pdf

Fairclough, N. (1995). Critical discourse analysis: The critical study of language. Londres: Pearson Education Limited.

Freire, P. (2006). Pedagogía de la autonomia: saberes necesarios para la práctica educativa. Madrid: Siglo XXI.

Freire, P. (1970). Pedagogía del oprimido (19a ed.). Madrid: Siglo XXI.

Freire, P. y Macedo, D. (1987). Literacy: Reading the word and the world. Londres: Bergin and Garvey.

Ghaemi, H. y Taherian, R. (2011). The role of critical thinking in EFL teachers' teaching success. Modern Journal of Applied Linguistics, 3(1), 8-22.

Giroux, H. A. (1997). Pedagogy and the politics of hope: Theory, culture, and schooling. A critical reader. Nueva York: West View Press.

Giroux, H. A. y McLaren, P. (1992). Writing from the margins: Geographies of identity, pedagogy, and power. Journal of Education, 174(1), 7-30. http://doi. org/10.1177/002205749217400103

Gómez, A., Puigvert, L., y Flecha, R. (2011). Critical communicative methodology: Informing real social transformation through research. Qualitative Inquiry, 17(3), 235-245. http://doi. org/10.1177/1077800410397802

Gounari, P. (2008). Devolviéndole lo crítico al lenguaje: una agenda crítica en la pedagogía de los idiomas. Revista Educación y Pedagogía, 51, 51-64.

Hawkins, M. y Norton, B. (2009). Critical language teacher education. En A. Burns y J. C. Richards (Eds.), The Cambridge guide to second language teacher education (pp. 30-39). Cambridge: Cambridge University Press. 
Jenkins, J. y Leung, C. (2014). English as a lingua franca. Nueva York, NY: John Wiley \& Sons, Inc. https://doi. org/10.1002/9781118411360.wbcla047

Jenson, J. D. (2011). Promoting Self-regulation and critical reflection through writing students' use of electronic portfolio. International Journal of ePortfolio, 1(1), 49-60.

Jiménez Catalán, R. M. (1997). La formación inicial del profesor de inglés de secundaria. Revista [Electrónica] Interuniversitaria de Formación del Profesorado, 1, 1-5.

Kachru, B. (1985). Standards, codification and sociolinguistic realm: the English language in the outer circle. En R. Quik y H. Widdowson (Eds.), English in the world: Teaching and learning the language and literature (pp. 11-30). Cambridge: Cup.

Kamhi-Stein, L. (1999). Preparing non-native professionals in TESOL: Implications for teacher education programs. En G. Braine (ed.), Non-native Educators in English language teaching (pp. 145-158). Nueva York: Routledge.

Kosnik, C., y Beck, C. (2009). Priorities in teacher education: the 7 key elements of pre-servicepreparation. Nueva York: Routledge. https://doi.org/10.4324/9780203870402

Kumaravadivelu, B. (2016). The decolonial option in English teaching: Can the subaltern act? TESOL Quarterly, 50(1), 66-85. https://doi.org/10.1002/tesq.202

Kumaravadivelu, B. (2006). TESOL methods: Changing tracks, challenging trends. TESOL Quarterly, 40, 5981. https://doi.org/10.2307/40264511

Lankshear, C. y McLaren, P. (Eds.) (1993). Critical literacy: Politics, praxis, and the postmodern. Nueva York: State University of New York Press.

Le Cornu, R., y Ewing, R. (2008). Reconceptualising professional experiences in pre-service teacher education... Reconstructing the past to embrace the future. Teaching and Teacher Education, 24(7), 1799-1812. https://doi.org/10.1016/j.tate.2008.02.008

Lee, I. (2007). Preparing pre-service English teachers for reflective practice. ELT Journal, 61(4), 321-329. https:// doi.org/10.1093/elt/ccm022

Ley Orgánica 2/2006, de 3 de mayo, de Educación. Boletín Oficial del Estado. Madrid, 4 de mayo de 2006, núm. 106, pp. 17158-17207.

Lin, A. M. Y. (2008). Cambios de paradigma en la enseñanza de inglés como lengua extranjera: el cambio crítico y más allá. Revista Educación y Pedagogía, 20(51), 11-23.

Lin, A. M. Y. (2004). Introducing a critical pedagogical curriculum: A feminist, reflexive account. En Norton, B., y Toohey, K. (Eds.). Critical pedagogies and language learning (pp. 271-290). Cambridge: Cup. https://doi. org/10.1017/CBO9781139524834.014

Llurda, E. (Ed.). (2006). Non-native language teachers: Perceptions, challenges and contributions to the profession. Nueva York: Springer Science \& Business Media.

Madrid, D. (2004). La formación inicial del profesorado de lengua extranjera. Profesorado. Revista de Curriculum y Formación del Profesorado, 8(1), 1-19.

Mahboob, A. (Ed.). (2010). The NNEST lens: Nonnative English speakers in TESOL. Newcastle upon Tyne: Cambridge Scholars Publishing.

Medgyes, P. (1992). Native or non-native: Who's worth more? ELT Journal, 46(4), 340-349. https://doi. org/10.1093/elt/46.4.340

Miller, E. R., Morgan, B. y Medina, A. L. (2017). Exploring language teacher identity work as ethical self-formation. The Modern Language Journal 101.S1: 91-105. https://doi.org/10.1111/modl.12371

Montero, L. y Vez, J. M. (1998). Processes of professionalisation and deprofessionalisation of teachers in Spain. TNTEE Publications, 1(2), 47-53.

Morgan, B. (2010). Fostering conceptual roles for change: Identity and agency in ESEA teacher preparation. Kritika Kultura, 15, 34-55.

Morgan, B. (2009). Fostering transformative practitioners for critical EAP: Possibilities and challenges. Journal of English for Academic Purposes, 8(2), 86-99. https://doi.org/10.1016/j.jeap.2008.09.001

Norton, B. (1997). Language, identity, and the ownership of English. TESOL Quarterly, 31(3), 409-429. https:// doi.org/10.2307/3587831

Norton, B. y Toohey, K. (2004). Critical pedagogies and language learning. Cambridge: Cambridge University Press. https://doi.org/10.1017/CBO9781139524834

Orden ECI/3858/2007, de 27 de diciembre, por la que se establecen los requisitos para la verificación de los títulos universitarios oficiales que habiliten para el ejercicio de las profesiones de Profesor de Educación Secundaria Obligatoria y Bachillerato, Formación Profesional y Enseñanzas de Idiomas. Boletín Oficial del Estado. Madrid, 29 de diciembre de 2007, núm. 312, pp. 53751- 53754 .

Orlova, N. (2009). Video recording as a stimulus for reflection in pre-service EFL Teacher Training. English Teaching Forum, 47(2), 30-35. us Department of State. Recuperado de: https://files.eric.ed.gov/fulltext/EJ923452.pdf 
Park, G. (2012). "I am never afraid of being recognized as an NNES": One Teacher's journey in claiming and embracing her nonnative-speaker identity. TESOL Quarterly, 46(1), 127-151. https://doi.org/10.1002/tesq.4

Pavlenko, A., y Blackledge, A. (Eds.). (2004). Negotiation of identities in multilingual contexts (vol. 45). Clevedon: Multilingual Matters. https://doi. org/10.21832/9781853596483

Pennycook, A. D. (2001). Critical applied linguistics: A critical introduction. Nueva York: Routledge. https://doi. org/10.4324/9781410600790

Pennycook, A. D. (1990). Critical pedagogy and second language education. System, 18(3), 303-314. https://doi. org/10.1016/0346-251X(90)90003-N

Philipson, R. (1992). Linguistic imperialism. Oxford: Oxford University Press.

Real Decreto 1393/2007, de 29 de octubre, por el que se establece la ordenación de las enseñanzas universitarias oficiales. Boletín Oficial del Estado. Madrid, 30 de octubre de 2007, núm. 260, pp. 44037-44048.

Richards, J. C., y Lockhart, C. (1991). Teacher development through peer observation. TESOL Journal, 1(2), 7-10.

Ruiz, I. G. y Marcos, A. R. (1994). Initial teacher education in Spain: A critical analysis. Journal of Education for Teaching, 20(3), 313-323. https://doi. org/10.1080/0260747940200305
Seidlhofer, B. (2013). Understanding English as a lingua franca (col. Oxford Applied Linguistics). Oxford: Oxford University Press. https://doi.org/10.1002 /9781405198431.wbeal0243

Shoffner, M. (2008). Informal reflection in pre-service teacher reflection. Reflective Practice, 9(2): 123-134. https://doi.org/10.1080/14623940802005392

Shor, I. (1992). Empowering education: Critical teaching for social change. Chicago: University of Chicago Press. https://doi.org/10.7208/chicago/97802261 47864.001.0001

Sierra, A. M. (2018). Changing teaching practices: The impact of a professional development program on an English teacher. Íkala, Revista de Lenguaje y Cultura, 23(1), 101-120. https://doi.org/10.17533/udea.ikala.v23n01a08

Siqueira, S. (2017). Intercultural language educators for an intercultural world: action upon reflection. Intercultural Education, 28(4), 390-407. https://doi.org/10. 1080/14675986.2017.1334396

Walters,L.M., Garii,B.y Walters, T.(2009).Learningglobally, teaching locally: incorporating international exchange and intercultural learning into pre-service teacher training. Intercultural Education, 20(1), 151-158. https://doi.org/10.1080/14675980903371050

Zabalza, M. (1991). Los diarios de clase. Barcelona: PpU.

How to reference this article: Barros-del Río, María Amor (2019). Cómo fomentar el espíritu crítico en los futuros docentes de inglés desde la formación: fundamentos y propuestas. Íkala, Revista de Lenguaje y Cultura, 24(3), 607-618. DoI: 10.17533/udea.ikala.v24n03a06 